\title{
Análise quantitativa de parâmetros biofísicos de bacia hidrográfica obtidos por sensoriamento remoto
}

\author{
Leidjane Maria Maciel de Oliveira( ${ }^{(1)}$, Suzana Maria Gico Lima Montenegro(1), Antônio Celso Dantas Antonino(1), \\ Bernardo Barbosa da Silva ${ }^{(1)}$, Célia Cristina Clemente Machado ${ }^{(1)}$ e Josiclêda Domiciano Galvíncio(1)
}

\begin{abstract}
(1)Universidade Federal de Pernambuco, Avenida Acadêmico Hélio Ramos, s/no, Cidade Universitária, CEP $50741-530$ Recife, PE. E-mail: leidjaneoliveira@hotmail.com, suzanam@ufpe.br, acda@ufpe.br, bbdasilva.ufpe@gmail.com, celiamachado.machado@gmail.com, josicleda@pq.cnpq.br
\end{abstract}

\begin{abstract}
Resumo - O objetivo deste trabalho foi avaliar quantitativamente os parâmetros biofísicos obtidos por sensoriamento remoto, para a área de abrangência da Bacia Hidrográfica do Rio Tapacurá, em Pernambuco. Utilizaram-se imagens do TM-Landsat 5 de 10/7/1989, 6/7/2005 e 29/8/2007. As imagens foram registradas pela correção geométrica polinomial de primeira ordem. Foram realizadas as etapas de calibração radiométrica, reflectância, albedo planetário e transmissividade e, subsequentemente, geraram-se cartas temáticas de albedo e de temperatura da superfície, e do índice de vegetação melhorado ("enhanced vegetation index", EVI). O albedo da superfície apresentou valores médios crescentes entre as imagens obtidas em 1989 e 2005, o que indica expansão territorial urbana. A imagem de 29/8/2007 mostrou maior temperatura da superfície, seguida das temperaturas mostradas nas imagens de 10/7/1989 e 6/7/2005, e os maiores valores foram os das malhas urbanas. A imagem de 1989 mostrou o maior valor médio de EVI, o que indica ter havido, naquela data, maior presença de vegetação.
\end{abstract}

Termos para indexação: albedo, EVI, expansão territorial urbana, monitoramento ambiental, temperatura da superfície, TM-Landsat 5.

\section{Quantitative analysis of biophysical parameters of watershed by remote sensing}

\begin{abstract}
The objective of this work was to quantitatively evaluate the biophysical parameters, obtained by remote sensing, for the range area of the Tapacurá River Basin, in Pernambuco state, Brazil. Landsat 5-TM images of $7 / 10 / 1989,7 / 6 / 2005$, and 8/29/2007 were used. The images were registered with a polynomial geometric correction of first order. Radiometric calibration, reflectance, transmittance and planetary albedo were calculated and, subsequently, thematic maps of surface albedo, surface temperature and enhanced vegetation index (EVI) were generated. The surface albedo showed increasing average values between the 1989 and 2005 images, which indicates the urban territorial expansion. The image of 8/29/2007 showed the highest land surface temperature, followed by the temperatures showed in the images of $7 / 10 / 1989$ and $7 / 6 / 2005$, and the highest values were those of the urban networks. The 1989 image showed the highest average value for EVI, which indicates that there was a higher vegetation presence in that date.
\end{abstract}

Index terms: albedo, EVI, urban territorial expansion, environmental monitoring, land surface temperature, TM-Landsat 5.

\section{Introdução}

O monitoramento de parâmetros biofísicos de uma bacia hidrográfica pode ajudar a estabelecer condições de uso e ocupação sustentáveis. Com o monitoramento, é possível diagnosticar alterações antrópicas e naturais na paisagem, e orientar o estabelecimento de políticas de reversão de um quadro de degradação ambiental (Cunha et al., 2012). A quantificação de parâmetros biofísicos - albedo, temperatura da superfície e alguns índices de vegetação - por meio de instrumentos convencionais, em escala regional, representaria um grande dispêndio de recursos materiais e humanos. Portanto, o uso do sensoriamento remoto orbital mostra-se como a opção economicamente mais viável em grandes áreas.

Estimativas do albedo da superfície por meio de imagens de satélites vêm sendo realizadas em grande escala. Giongo et al. (2010) avaliaram o albedo de Santa Rita do Passa Quatro, SP, por meio de imagens do TM-Landsat 5 e obtiveram resultados concordantes com medições realizadas em duas torres

Pesq. agropec. bras., Brasília, v.47, n.9, p.1209-1217, set. 2012 
micrometeorológicas, localizadas na área estudo - uma sobre cerrado e outra em plantio de cana-de-açúcar. Silva et al. (2010) identificaram alterações climáticas decorrentes de mudanças no uso da terra mediante sensoriamento remoto, nas Bacias do Rio Mogi-Guaçu, $\mathrm{SP}$, e do Baixo Jaguaribe, CE, e verificaram boa concordância entre as imagens obtidas e as medições micrometeorológicas existentes nas áreas do estudo. Bezerra et al. (2011) constataram haver interferência atmosférica no cômputo do albedo, ao investigar alteração em diversos biomas na divisa de Pernambuco e Ceará, por meio de imagens TM-Landsat 5, e concluíram que áreas com menor cobertura vegetativa foram mais afetadas pelos impactos numéricos das correções atmosféricas.

Outros parâmetros biofísicos empregados no monitoramento ambiental são a temperatura da superfície $\left(\mathrm{T}_{\text {sup }}\right)$ e alguns índices de vegetação. $\mathrm{A} \mathrm{T}_{\text {sup }}$ está envolvida no balanço de energia, evaporação e transpiração da vegetação, bem como em processos de desertificação; o que a torna adequada para uso como indicador de degradação terrestre e de mudança climática. Araújo \& Di Pace (2010) obtiveram a $\mathrm{T}_{\text {sup }}$ em Alagoas, com imagens do TM-Landsat 5, e constataram que as maiores temperaturas encontramse dentro das malhas urbanas, decorrentes da impermeabilização do solo e outras fontes de calor antropogênicas. Gusso et al. (2007) mapearam a $\mathrm{T}_{\text {sup }}$ no Rio Grande do Sul, com o emprego de imagens AVHRR, e correlacionaram a $\mathrm{T}_{\text {sup }}$ à temperatura do ar medida em diferentes estações meteorológicas daquele estado. Silva et al. (2011) pesquisaram vários tipos de cobertura do solo, com imagens do TM-Landsat 5, na Paraíba, e constataram que a presença de áreas irrigadas contribuiu substancialmente para reduzir a $\mathrm{T}_{\text {sup }} \mathrm{e}$, consequentemente, para diminuir a temperatura do ar, com efeitos sobre o clima local.

As mudanças do uso e ocupação do solo podem ser detectadas por meio de índices de vegetação derivados de imagens orbitais. Adami et al. (2008) conseguiram distinguir áreas permanentemente alagadas, inundáveis e secas no bioma Pantanal, por meio de imagens do sensor Modis (moderate resolution imaging spectroradiometer), com uso do índice de vegetação melhorado (enhanced vegetation index, EVI), conjugados a dados de precipitação e de altimetria. Formigoni et al. (2011) analisaram o comportamento temporal do EVI gerado com imagens Modis, em diferentes tipos de vegetação do Nordeste brasileiro, no período de 2000 a 2006, e constataram que o EVI apresentou sensibilidade aos diferentes tipos de vegetação existentes na região.

A Bacia Hidrográfica do Rio Tapacurá tem grande importância na gestão dos recursos hídricos enaturais do Estado de Pernambuco, pois gera mais de $25 \%$ da água consumida na região metropolitana do Recife. Ela está presente em seis municípios, apresenta multiplicidade de conflitos de uso da água (abastecimento público, irrigação, uso industrial e outros) e possibilita o controle de inundações, por meio da barragem do rio Tapacurá, construída em meados da década de 1970. A quantificação de parâmetros biofísicos pode ser empregada no diagnóstico e monitoramento ambiental e na identificação de possíveis alterações do uso e ocupação do solo desta bacia.

O objetivo deste trabalho foi avaliar quantitativamente os parâmetros biofísicos - albedo, $\mathrm{EVI}_{\text {e }} \mathrm{T}_{\text {sup }}-$, obtidos por meio de técnicas de sensoriamento remoto orbital, para a área de abrangência da Bacia do Rio Tapacurá, PE.

\section{Material e Métodos}

A área de estudo compreende a Bacia Hidrográfica do Rio Tapacurá, sub-bacia do Rio Capibaribe, PE, integrante da Rede de Hidrologia do Semiárido (REHISA), com área de drenagem de $470,50 \mathrm{~km}^{2}$. A região localiza-se entre $07^{\circ} 58^{\prime}$ e $08^{\circ} 13^{\prime} \mathrm{S}$ e $35^{\circ} 05^{\prime}$ e $35^{\circ} 0^{\prime} \mathrm{W}$. O relevo da bacia é constituído, em sua maior parte, por colinas e cristas. A temperatura mensal média do ar varia de 23 a $27^{\circ} \mathrm{C}$, a umidade relativa do ar é superior a $70 \%$, e a precipitação média anual situa-se entre 850 e $1.600 \mathrm{~mm}$. Apresenta áreas tanto da Zona da Mata Úmida como do Agreste Subúmido, com presença de remanescentes da Mata Atlântica e alguns remanescentes de Caatinga (Braga, 2001; Duarte et al., 2007).

Foram adquiridas, junto ao Instituto Nacional de Pesquisas Espaciais (Inpe), três imagens do mapeador temático do satélite Landsat 5, órbita 214 e ponto 66 , referentes a: 10 de julho de 1989, 6 de julho de 2005 e 29 de agosto de 2007. Essas imagens foram empilhadas, banda à banda e, em seguida, registradas pela correção geométrica polinomial de primeira ordem, com seis pontos de controle e erro sempre inferior a um pixel. Utilizou-se uma imagem de referência ortorretificada, obtida na página htpp://www.landsat.org. Assim, foram geradas cartas temáticas de albedo da superfície, EVI e 
temperatura da superfície. O modelo digital de elevação (MDE) das cenas SB25YC e SB25VA foi utilizado na geração do mapa de altitude e foi reamostrado para 30x30 m, de modo a propiciar estimativa da pressão atmosférica em cada pixel.

As variáveis biofísicas - albedo, temperatura da superfície e EVI - foram obtidas em várias etapas, que se iniciaram pela calibração radiométrica, ou seja, pela transformação do nível de cinza de cada pixel em radiância espectral $\left(\mathrm{L}_{\lambda_{\mathrm{i}}}\right)$, por meio da equação, $\mathrm{L}_{\lambda \mathrm{i}}=\mathrm{a}_{\mathrm{i}}+\left(\mathrm{b}_{\mathrm{i}}+\mathrm{a}_{\mathrm{i}}\right) / 255 \mathrm{x} \mathrm{ND}$, proposta por Markham \& Baker (1987), em que: a e b são radiâncias espectrais mínima e máxima $\left(\mathrm{Wm}^{-2} \mathrm{sr}^{-1} \mu \mathrm{m}^{-1}\right)$, obtidas de acordo com Chander et al. (2007); ND é a intensidade do pixel (número inteiro entre 0 e 255); e i representa cada banda do TM-Landsat 5 .

A reflectância $\left(\rho_{\lambda_{i}}\right)$ corresponde à razão entre $o$ fluxo de radiação refletida por cada banda e o fluxo de radiação incidente, e foi obtida conforme Silva et al. (2005b) e Bezerra et al. (2011), pela equação $\rho_{\lambda_{\mathrm{i}}}=\pi \mathrm{L}_{\lambda_{\mathrm{i}}} /\left(\mathrm{k}_{\lambda_{\mathrm{i}}} \times \cos \mathrm{Zdr}\right)$, em que: $\mathrm{L}_{\lambda \mathrm{i}}\left(\mathrm{Wm}^{-2} \mathrm{sr}^{-1} \mu \mathrm{m}^{-1}\right)$ é a radiância espectral de cada banda; $\mathrm{k}_{\lambda \mathrm{i}}\left(\mathrm{Wm}^{-2} \mu \mathrm{m}^{-1}\right)$ é a irradiância solar espectral de cada banda, no topo da atmosfera, obtida de acordo com Chander \& Markham (2003); Z é o ângulo zenital solar; e $d_{r}$ é o quadrado da razão entre a distância média Terra-Sol $\left(\mathrm{r}_{\mathrm{o}}\right)$ e a distância Terra-Sol (r), em dado dia do ano.

O albedo planetário $\left(\alpha_{\text {toa }}\right)$ para cada banda, isto é, o albedo não ajustado às interferências atmosféricas, foi obtido por meio de combinação linear entre as reflectâncias monocromáticas, por, $\alpha_{\text {toa }}=0,293 \rho_{1}+$ $0,274 \rho_{2}+0,233 \rho_{3}+0,155 \rho_{4}+0,032 \rho_{5}+0,012 \rho_{7}$, em que: $\rho_{1}, \rho_{2}, \rho_{3}, \rho_{4}, \rho_{5}$ e $\rho_{7}$ foram os albedos planetários associados às bandas $1,2,3,4,5$ e 7, respectivamente; e cada fator $(0,293, \ldots, 0,012)$ correspondeu à razão entre a irradiância solar, no domínio de cada banda, pela somatória de todas essas irradiâncias (Silva et al., 2005b).

A transmissividade atmosférica $\left(\tau_{\mathrm{sw}}\right)$ foi obtida conforme Allen et al. (2007), em função da pressão atmosférica e da água precipitável por meio da equação $\tau_{\mathrm{sw}}=0,35+0,627 \exp \left[-0,00146 \mathrm{P} /\left(\mathrm{K}_{\mathrm{t}} \cos \mathrm{Z}\right)-\right.$ $\left.0,075(\mathrm{~W} / \cos Z)^{0,4}\right]$, em que: $\mathrm{Z}$ é o ângulo zenital solar; $\mathrm{K}_{\mathrm{t}}$ é o coeficiente de turbidez da atmosfera $\left(\mathrm{K}_{\mathrm{t}}=1\right.$, para céu claro e limpo); P é a pressão atmosférica $(\mathrm{kPa})$; e $\mathrm{W}$ é a água precipitável $(\mathrm{mm})$, calculadas conforme Garrison \& Adler (1990), pelas equações, $\mathrm{P}=101,3\left(\mathrm{~T}_{\mathrm{a}}-0,0065 \mathrm{~h}\right) / \mathrm{T}_{\mathrm{a}}$ e $\mathrm{W}=0,14 \mathrm{e}_{\mathrm{a}} \mathrm{P}+2,10$, respectivamente, em que: $T_{a}$ é a temperatura do ar ( Kelvin); h (m) é a altitude da área em estudo, pelo modelo digital de elevação (MDE); e $e_{a}$ é a pressão atual de vapor de água $(\mathrm{kPa})$, que é função da umidade relativa do ar $(\%)$ e pressão de saturação $(\mathrm{KPa})$ à temperatura do ar.

O albedo da superfície $(\alpha)$ ou albedo corrigido dos efeitos atmosféricos foi obtido pela equação, $\alpha=\left(\alpha_{\text {toa }}-\alpha_{\mathrm{p}}\right) / \tau_{\mathrm{sw}}{ }^{2}$ (Bastiaanssen et al., 1998), em que: $\alpha_{\text {toa }}$ é o albedo planetário; $\alpha_{\mathrm{p}}$ é a reflectância atmosférica, considerada nesta pesquisa igual a 0,03 , conforme Bastiaanssen (2000); e $\tau_{\mathrm{sw}}$ é a transmitância atmosférica.

Para a determinação da temperatura da superfície ( $\mathrm{T}_{\text {sup, }}$ em ${ }^{\circ}$ Kelvin), relacionou-se a radiância $\left(\mathrm{L}_{\lambda \mathrm{i}}\right)$ da banda termal do TM-Landsat 5 (banda 6) e a emissividade $\left(\varepsilon_{\mathrm{NB}}\right)$ no domínio espectral dessa banda (Narrow Band), por meio da equação $\mathrm{T}_{\text {sup }}=\mathrm{K}_{2} / \ln \left(\varepsilon_{\mathrm{NB}} \mathrm{K}_{1} / \mathrm{L}_{\lambda 6}+1\right)$, conforme Allen et al. (2007), em que: $\mathrm{K}_{1}=607,76\left(\mathrm{Wm}^{-2} \mathrm{sr}^{-1} \mu \mathrm{m}^{-1}\right) \mathrm{e}$ $\mathrm{K}_{2}=1.260,56(\mathrm{~K})$ são constantes de calibração específicas do TM-Landsat 5.

$\mathrm{Na}$ determinação da emissividade $\left(\varepsilon_{\mathrm{NB}}\right)$, empregouse a expressão $\varepsilon_{\mathrm{NB}}=0,97+0,0033 \mathrm{IAF}$, em que: IAF é o índice de área foliar $\left(\mathrm{m}^{2} \mathrm{~m}^{-2}\right)$, obtido com base no índice de vegetação ajustado para efeitos do solo (Savi) que é função da reflectância do infravermelho próximo e vermelho. Esta equação tem validade para $\mathrm{IAF}<3$ e, no caso em que o $\mathrm{IAF} \geq 3$, considera-se $\varepsilon_{\mathrm{NB}}=0,98$ e, para corpos de água $\varepsilon_{\mathrm{NB}}=0,99$.

$\mathrm{O}$ índice de vegetação melhorado (EVI) foi desenvolvido para melhorar a sensibilidade para $\mathrm{o}$ sinal da vegetação verde, o que minimiza os efeitos do solo e da atmosfera. A equação que define este parâmetro foi desenvolvida por Huete et al. (1997) como EVI $=\mathrm{G}\left(\rho_{\mathrm{IV}}-\rho_{\mathrm{V}}\right) /\left(\mathrm{L}+\rho_{\mathrm{IV}}+\mathrm{C}_{1} \rho_{\mathrm{V}}-\mathrm{C}_{2} \rho_{\text {azul }}\right)$, em que: $\mathrm{G}$ é o fator de ganho $(2,5)$; $\rho_{\mathrm{IV}}$ é a reflectância do infravermelho próximo; $\rho_{\mathrm{V}}$ é a reflectância do vermelho; $\rho_{\text {azul }}$ é a reflectância do azul; $\mathrm{L}$ é o fator de ajuste para solos $(1,0)$; e $\mathrm{C}_{1}$ e $\mathrm{C}_{2}$ descrevem o uso da banda azul, para correção da banda vermelha quanto ao espalhamento atmosférico por aerossóis, com valores 6,0 e 7,5, respectivamente (Huete et al., 1997). As bandas reflectivas empregadas no cômputo do EVI foram corrigidas atmosfericamente, conforme os procedimentos do Metric propostos por Allen et al. (2007).

Os dados de precipitação e temperatura do ar (Figura 1) da Bacia do Rio Tapacurá foram obtidos no Instituto de Tecnologia de Pernambuco (Itep). 
Para o ano de 1989, os dados corresponderam à estação convencional, pois a plataforma de coleta de dados (PCD) só foi implantada em 1999. Ademais, o

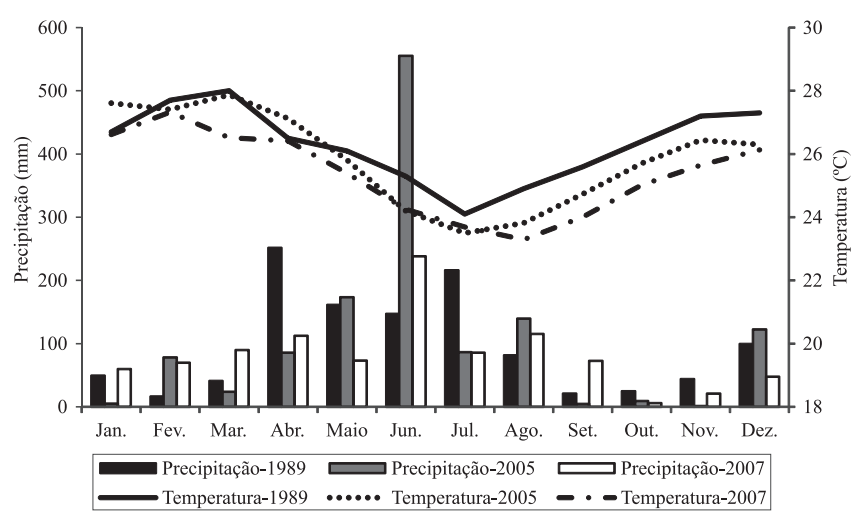

Figura 1. Precipitação mensal e temperatura média do ar, de 1989, 2005 e 2007, na Bacia do Rio Tapacurá, PE. horário sinóptico das 12 h (tempo médio de Greenwich) correspondente ao horário local de $9 \mathrm{~h}$, portanto, muito próximo ao horário de passagem do Landsat 5. No ano de 2005, foram detectadas falhas nas medições de temperatura do ar. Assim, optou-se por fazer uma junção da PCD da cidade de Palmares - pertencente à mesma região de desenvolvimento (Mata Sul) - a microrregiões vizinhas (Vitória de Santo Antão e Mata Meridional Pernambucana) com a mesma faixa de variação da temperatura do ar média anual (Silva, 2006). Para o ano de 2007, os dados corresponderam à PCD existente e em operação.

\section{Resultados e Discussão}

A variação espaço-temporal do albedo da superfície na Bacia do Rio Tapacurá, nas datas de 10/7/1989, 6/7/2005 e 29/8/2007 (Figura 2), indica pequenas
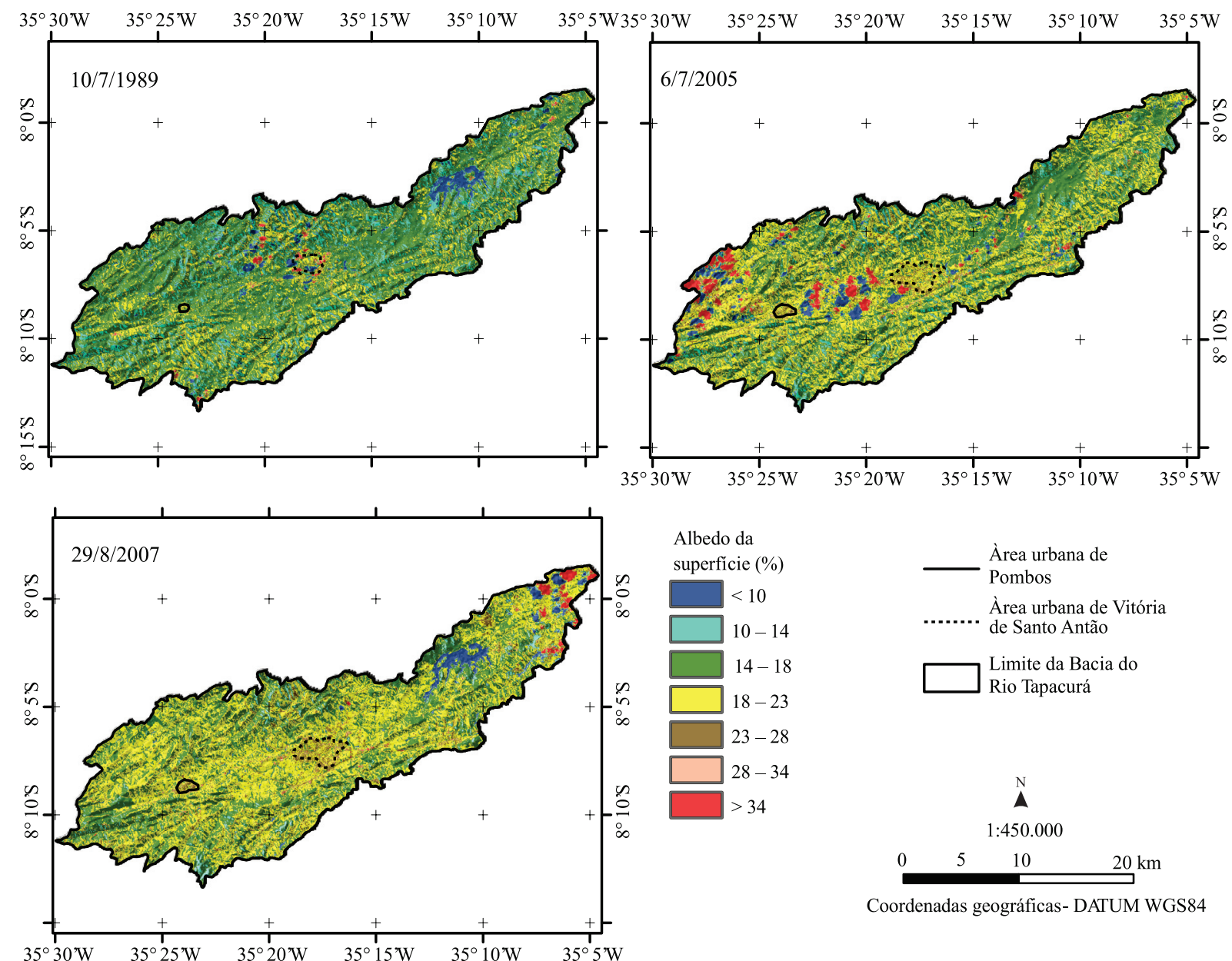

Figura 2. Distribuição espacial do albedo da superfície, estimada por meio da banda TM-Landsat 5, em 10/7/1989, 6/7/2005 e 29/8/2007, na região da Bacia do Rio Tapacurá, PE. 
áreas com albedo maior que 34\% (nuvens), seguidas imediatamente de áreas com albedo menor que $10 \%$ (sombra das nuvens).

Houve aumento gradativo do albedo na sequência das imagens, principalmente nas áreas urbanas da área de estudo, onde o albedo extrapolou de uma faixa de 14-18\% (1989) para 23-28\% (2005 e 2007). Este aumento está relacionado à expansão territorial urbana dos municípios que integram os limites da bacia, detectada mais nitidamente de 1989 para 2005. As precipitações também podem afetar os valores do albedo, pois promovem redução da radiação refletida pelo solo e, no caso da Caatinga, aumentam o índice de área foliar da vegetação após um evento chuvoso. Conforme Figura 2, a imagem de 10/7/1989 foi a mais afetada pela chuva (Figura 1) e registrou o menor valor médio de albedo $(\alpha=16,1 \%)$ (Tabela 1$)$.

As alterações registradas no albedo estão associadas às mudanças no uso e ocupação do solo, que podem ter contribuído para afetar o clima localmente. Silva et al. (2010) pesquisaram as alterações climáticas decorrentes de mudanças do uso da terra na Bacia do Rio Jaguaribe, no Ceará, e encontraram albedo entre 14 e $18 \%$, áreas isoladas com variação de $22-26 \%$ e locais com possível presença de bancos de areia, com albedo superior a 28\%. Oliveira et al. (2010) analisaram o albedo da superfície da Bacia Hidrográfica do Rio Moxotó, PE, e concluíram que áreas com desenvolvimento de ações antrópicas e solos expostos apresentaram os valores mais elevados do albedo da superfície (albedo $>30 \%$ ).

No tocante à distribuição do pixel que determina as classes do albedo, pode-se observar que, ao longo do período analisado, a faixa do albedo de $14-18 \%$ apresentou diminuição ao longo do período investigado, enquanto a classe de $18-23 \%$ teve aumento expressivo (Tabela 1). Os valores médios do albedo, nas imagens de 1989, 2005 e 2007, revelaram aumento entre as imagens dos anos de 1989 e 2005. Esse aumento pode ser explicado pela diminuição de áreas vegetadas, representadas pela classe de $14-18 \%$ e aumento do albedo provocado por áreas com maior exposição dos solos, que estariam associadas à classe de maiores valores (18-23\%).

O menor coeficiente de variação $(\mathrm{CV})$ foi observado para a imagem obtida em 1989, o que confirmou maior homogeneidade do albedo, em detrimento do que foi observado nas imagens obtidas em 2005 e 2007, que apresentaram menor homogeneidade. Essa variação na homogeneidade entre os anos decorreu de ações antrópicas, como a multiplicidade do uso do solo e aumento da expansão territorial urbana.

Houve brusca mudança de classe de $\mathrm{T}_{\text {sup }}$ entre $10 / 7 / 1989\left(18-23^{\circ} \mathrm{C}\right)$ e $29 / 8 / 2007\left(23-30^{\circ} \mathrm{C}\right)$, e $\mathrm{T}_{\text {sup }}$ maior que $30^{\circ} \mathrm{C}$ nos centros urbanos das cidades de Pombos e Vitória de Santo Antão, em 29/8/2007 (Figura 3). Essas alterações dos padrões da $\mathrm{T}_{\text {sup }}$ corroboraram as alterações nos padrões de albedo, analisadas anteriormente.

Tabela 1. Distribuição percentual das classes de albedo da superfície, temperatura da superfície e do EVI na Bacia do Rio Tapacurá, PE, com respectivos valores estatísticos, e valores de precipitação $(\mathrm{mm})$ de junho até a data da imagem e nos 10 dias anteriores à imagem.

\begin{tabular}{|c|c|c|c|}
\hline \multirow[t]{2}{*}{ Classes } & \multicolumn{3}{|c|}{ Distribuição percentual } \\
\hline & $10 / 7 / 1989$ & $6 / 7 / 2005$ & $29 / 8 / 2007$ \\
\hline \multicolumn{4}{|l|}{ Albedo (\%) } \\
\hline$<10$ & 3,28 & 3,11 & 2,29 \\
\hline $10-14$ & 15,22 & 7,17 & 3,54 \\
\hline $14-18$ & 60,86 & 44,44 & 43,33 \\
\hline $18-23$ & 18,94 & 37,93 & 45,01 \\
\hline $23-28$ & 0,91 & 3,79 & 4,04 \\
\hline $28-34$ & 0,35 & 0,86 & 0,69 \\
\hline$>34$ & 0,44 & 2,70 & 1,10 \\
\hline Valor Médio (\%) & 16,1 & 18,8 & 18,5 \\
\hline Desvio-padrão & 0,035 & 0,098 & 0,058 \\
\hline Coeficiente de variação (\%) & 21,74 & 52,13 & 31,35 \\
\hline \multicolumn{4}{|l|}{ Temperatura $\left({ }^{\circ} \mathrm{C}\right)$} \\
\hline$<18$ & 0,79 & 9,92 & 0,65 \\
\hline $18-21$ & 72,32 & 86,11 & 1,40 \\
\hline $21-23$ & 26,86 & 3,73 & 15,92 \\
\hline $23-25$ & 0,03 & 0,24 & 49,87 \\
\hline $25-27$ & 0,00 & 0,00 & 24,55 \\
\hline $27-30$ & 0,00 & 0,00 & 7,05 \\
\hline$>30$ & 0,00 & 0,00 & 0,56 \\
\hline Valor Médio & 20,49 & 19,14 & 24,39 \\
\hline Desvio-padrão & 0,770 & 1,313 & 1,899 \\
\hline Coeficiente de variação (\%) & 3,76 & 6,86 & 7,79 \\
\hline \multicolumn{4}{|l|}{ EVI } \\
\hline$<0,00$ & 1,59 & 2,37 & 1,40 \\
\hline $0,00-0,20$ & 2,74 & 3,53 & 3,77 \\
\hline $0,20-0,40$ & 6,23 & 11,39 & 16,65 \\
\hline $0,40-0,60$ & 40,82 & 34,72 & 62,33 \\
\hline $0,60-0,70$ & 30,76 & 23,22 & 13,36 \\
\hline $0,70-0,80$ & 14,09 & 20,35 & 2,33 \\
\hline$>0,80$ & 3,77 & 4,42 & 0,16 \\
\hline Valor Médio & 0,570 & 0,552 & 0,479 \\
\hline Desvio-padrão & 0,173 & 0,198 & 0,145 \\
\hline Coeficiente de variação (\%) & 30,35 & 35,86 & 30,27 \\
\hline Precipitação & 206,20 & 568,70 & 435,70 \\
\hline Precipitação 10 dias antes & 66,70 & 33,80 & 15,50 \\
\hline
\end{tabular}


Pela imagem de 6/7/2005, praticamente toda a bacia hidrográfica apresentou temperatura da superfície menor que $21^{\circ} \mathrm{C}$, exceto nas áreas urbanas (Pombos e Vitória de Santo Antão). Justifica-se este fato pela influência do período chuvoso (Figura 1), já que no mês de junho choveu quase todos os dias, com um total de $568,7 \mathrm{~mm}$ de precipitação entre 1/6/2005 e 6/7/2005. O suprimento hídrico no período favoreceu a diminuição da temperatura da superfície. Este mesmo comportamento foi constatado por Silva et al. (2005a), em estudo realizado pelo Projeto Senador Nilo Coelho em PE e BA. Quando estes autores compararam os anos estudados, constataram que o ano de 2000 apresentou maior índice pluvial, com menores $\mathrm{T}_{\text {sup }}\left(23,1\right.$ a $\left.37,5^{\circ} \mathrm{C}\right)$, enquanto o ano de 2001 apresentou menor índice pluvial, com maiores $\mathrm{T}_{\text {sup }}\left(24,7\right.$ a $\left.39,9^{\circ} \mathrm{C}\right)$.

Nas três imagens avaliadas, os maiores valores de $T_{\text {sup }}$ foram encontrados dentro da malha urbana, resultado que corrobora os de Araújo \& Di Pace (2010), em estudo realizado na cidade de Maceió, AL. Ao espacializar a temperatura da superfície da cidade do Recife, Moreira \& Galvíncio (2007) também constataram que áreas com maior adensamento - construções verticais, horizontais, concentração de veículos -, associada à baixa presença arbórea, contribuíram para o aumento da temperatura local.

Observa-se que, entre as imagens de 6 de julho de 2005 e 29 de agosto de 2007, foi bastante acentuada a contribuição percentual da distribuição do pixel na classe da $\mathrm{T}_{\text {sup }}$ de $18-21^{\circ} \mathrm{C}$ (Tabela 1 ), que resulta do fator pluviometria, mas também da contribuição associada à radiação solar incidente, maior em 29 de agosto de 2007. Houve, para esta imagem, aumento expressivo nas classes de maior temperatura, às dos anos anteriores. $\mathrm{O}$ aumento da $\mathrm{T}_{\text {sup }}$ média, observada em 29 de agosto de 2007, foi consequência também da expansão urbana, o que aumentou seu desvio-padrão e coeficiente de variação (Figura 3 e Tabela 1).
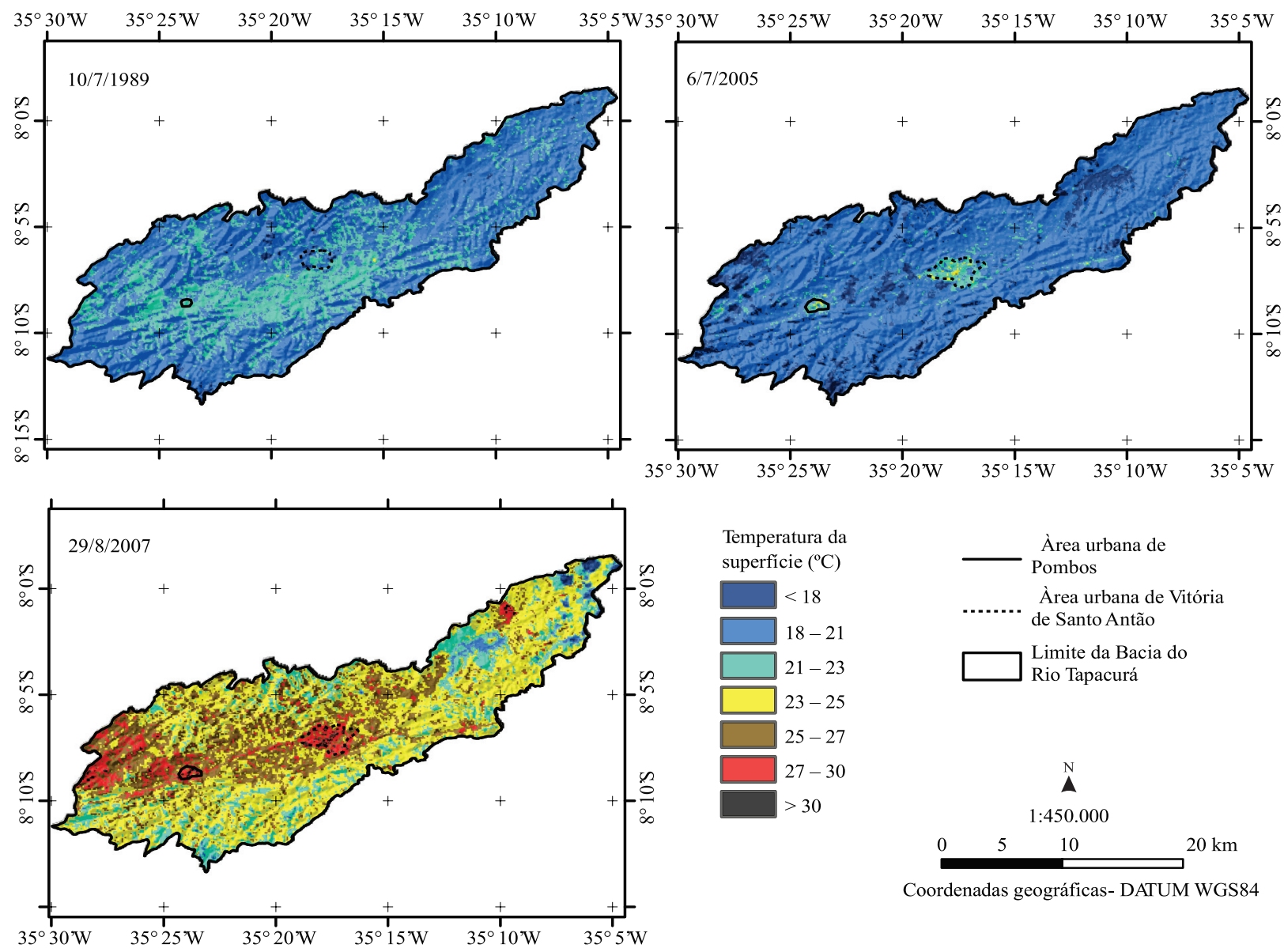

Figura 3. Distribuição espacial da temperatura da superfície, estimada por meio da banda TM-Landsat 5, em 10/7/1989, 6/7/2005 e 29/8/2007, na região da Bacia do Rio Tapacurá, PE. 
$\mathrm{O}$ albedo da superfície e a $\mathrm{T}_{\text {sup }}$ apresentaram maior homogeneidade na imagem de 1989, comprovada pelo menor coeficiente de variação $(\mathrm{CV})$ entre as três datas estudadas. Entretanto, a maior média da $\mathrm{T}_{\text {sup }}$ correspondeu à imagem do ano de 2007, e a maior média do albedo foi no ano de 2005 (Tabela 1).

Nas imagens de 10/7/1989 e 6/7/2005 (Figura 4), o EVI $>0,80$ esteve mais destacado entre as coordenadas $8^{\circ} 3^{\prime} \mathrm{S}$ e $35^{\circ} 12^{\prime} \mathrm{W}$, região correspondente à área inundável do Reservatório Tapacurá. O contorno da referida área inundável apresentou maior abrangência na imagem do dia 6 de julho de 2005, em razão das maiores precipitações ocorridas entre junho e agosto (Figura 1). O comportamento dos eventos chuvosos foi condizente com a análise da variabilidade espaço-temporal realizada por Silva et al. (2010a) na Bacia do Rio Tapacurá, no período de 1970 a 2000. Os autores constataram que nos meses de março a julho ocorrem $60 \%$ da precipitação total anual e que o período seco ocorre entre agosto e fevereiro.

O EVI foi desenvolvido para aperfeiçoar o sinal da vegetação que, por sua vez, depende da umidade disponível no solo. Observa-se que a imagem de 1989 mostrou maior valor médio de EVI $(0,57)$ (Tabela 1$)$, mesmo tendo apresentado a menor precipitação de junho até a data da imagem (206,2 mm). Entretanto, nota-se que, nos 10 dias antecedentes à imagem, esse ano apresentou maior índice pluvial do que os outros anos $(66,7 \mathrm{~mm})$ e, ainda, neste mesmo período de 10 dias, somente em dois não ocorreram eventos chuvosos. Esta relação entre EVI e a precipitação foi enfatizada por Becerra et al. (2009), em estudo sobre a relação entre a vegetação e a precipitação na região do Cerrado, por meio dos índices de vegetação NDVI e EVI. Os autores observaram que o período da pesquisa com menor precipitação apresentou os menores valores de EVI.
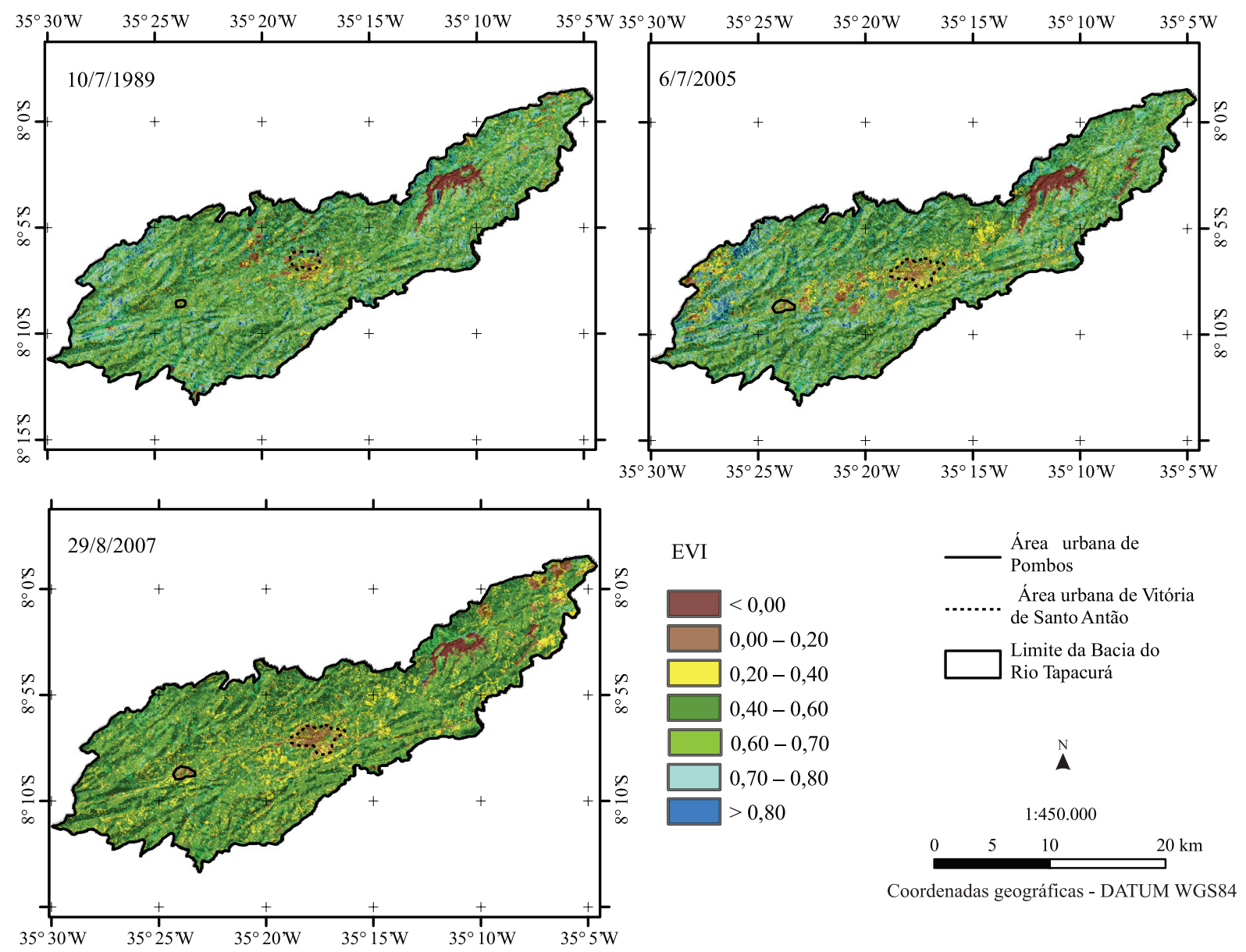

Figura 4. Distribuição espacial do índice de vegetação melhorado (EVI), estimado por meio da banda TM-Landsat 5, em 10/7/1989, 6/7/2005 e 29/8/2007, na região da Bacia do Rio Tapacurá, PE. 


\section{Conclusões}

1. O aumento do albedo e da temperatura da superfície, bem como a redução do índice de vegetação melhorado, observados por meio de sensoriamento remoto orbital, permitem identificar alterações do uso e ocupação do solo.

2. O albedo e o índice de vegetação melhorado identificam mais claramente a variabilidade do uso e ocupação do solo na Bacia Hidrográfica do Rio Tapacurá, PE.

\section{Agradecimentos}

À Financiadora de Estudos e Projetos e ao Conselho Nacional de Desenvolvimento Científico e Tecnológico, pelo apoio financeiro e concessão de bolsas; ao Instituto de Tecnologia de Pernambuco, pelo fornecimento do banco de dados; e ao Instituto Nacional de Pesquisas Espaciais, pelo fornecimento das imagens do TM-Landsat 5.

\section{Referências}

ADAMI, M.; FREITAS, R.M. de; PANDOVANI, C.R.; SHIMABUKURO, Y.E.; MOREIRA, M.A. Estudo da dinâmica espaço-temporal do bioma Pantanal por meio de imagens MODIS. Pesquisa Agropecuária Brasileira, v.43, p.1371-1378, 2008.

ALLEN, R.G.; TASUMI, M.; TREZZA, R. Satellite-based energy balance for mapping evapotranspiration with internalized calibration (Metric) - model. Journal of Irrigation and Drainage Engineering, v.133, p.380-394, 2007.

ARAÚJO, T.L.; DI PACE, F.T. Valores instantâneos da temperatura da superfície terrestre na Cidade de Maceió-AL utilizando imagens do satélite TM/Landsat 5. Revista Brasileira de Geografia Física, v.3, p.104-111, 2010.

BASTIAANSSEN, W.G.M. SEBAL-based sensible and latent heat fluxes in the irrigated Gediz Basin, Turkey. Journal of Hydrology, v.229, p.87-100, 2000.

BASTIAANSSEN, W.G.M.; MENENTI, M.; FEDDES, R.A.; HOLSLAG, A.A.M. A remote sensing surface energy balance algorithm for land (SEBAL) - 1. Formulation. Journal of Hydrology, v.212-213, p.198-212, 1998.

BECERRA, J.A.B.; SHIMABUKURO, Y.E.; ALVALÁ, R.C. dos S. Relação do padrão sazonal da vegetação com a precipitação na região de Cerrado da Amazônia Legal, usando índices espectrais de vegetação. Revista Brasileira de Meteorologia, v.24, p.125-134, 2009.

BEZERRA, M.V.C.; SILVA, B.B. da; BEZERRA, B.G. Avaliação dos efeitos atmosféricos no albedo e NDVI obtidos com imagens de satélite. Revista Brasileira de Engenharia Agrícola e Ambiental, v.15, p.709-717, 2011.
BRAGA, R.A.P. Gestão ambiental da Bacia do Rio Tapacurá: plano de ação. Recife: UFPE, 2001. 101p.

CHANDER, G.; MARKHAM, B. Revised Landsat-5 TM radiometric calibration procedures and postcalibration dynamic ranges. IEEE Transactions on Geoscience and Remote Sensing, v.41, p.2674-2677, 2003.

CHANDER, G.; MARKHAM, B.L.; BARSI, J.A. Revised Landsat-5 thematic mapper radiometric calibration. IEEE Geoscience and Remote Sensing Letters, v.4, p.490-494, 2007.

CUNHA, J.E. de B.L.; RUFINO, I.A.A.; SILVA, B.B. da; CHAVES, I. de B. Dinâmica da cobertura vegetal para a Bacia de São João do Rio do Peixe, PB, utilizando-se sensoriamento remoto. Revista Brasileira de Engenharia Agrícola e Ambiental, v.16, p.539-548, 2012.

DUARTE, C.C.; GALVÍNCIO, J.D.; CORRÊA, A.C. de B.; ARAÚJO, M. do S.B. de. Análise fisiográfica da Bacia Hidrográfica do Rio Tapacurá-PE. Revista de Geografia, v.24, p.50-64, 2007.

FORMIGONI, M. de H.; XAVIER, A.C.; LIMA, J.S. de S. Análise temporal da vegetação na região do Nordeste através de dados EVI do MODIS. Ciência Florestal, v.21, p.1-8, 2011.

GARRISON, J.D.; ADLER, G.P. Estimation of precipitable water over the United State for application to the division of solar radiation into its direct and diffuse components. Solar Energy, V.44, p.225-241, 1990.

GIONGO, P.R.; MOURA, G.B. de A.; SILVA, B.B. da; ROCHA, H.R. da; MEDEIROS, S.R.R. de; NAZARENO, A.C. Albedo à superfície a partir de imagens Landsat 5 em áreas de cana-de-açúcar e cerrado. Revista Brasileira de Engenharia Agrícola e Ambiental, v.14, p.279-287, 2010.

GUSSO, A.; FONTANA, D.C.; GONÇALVES, G.A. Mapeamento da temperatura da superfície terrestre com o uso do sensor AVHRR/ NOAA. Pesquisa Agropecuária Brasileira, v.42, p.231-237, 2007.

HUETE, A.R.; LIU, H.Q.; BATCHIL, Y.K.; LEEUWEN, W. van. A comparison of vegetation indices over a global set of TM images for EOS-MODIS. Remote Sensing of Environment, v.59, p.440-451, 1997.

MARKHAM, B.L.; BARKER, J.L. Thematic mapper bandpass solar exoatmospheric irradiances. International Journal of Remote Sensing, v.8, p.517-523, 1987.

MOREIRA, E.B.M.; GALVÍNCIO, J.D. Espacialização das temperaturas à superfície na cidade do Recife, utilizando imagens TM - LANNDSAT 7. Revista de Geografia, v.24, p.101-115, 2007.

OLIVEIRA, T.H. de; MACHADO, C.C.C.; SILVA, J.S. e; GALVÍNCIO, J.D.; PIMENTEL, R.M. de M.; SILVA, B.B. da. Índice de umidade (NDWI) e análise espaço-temporal do albedo da superfície da bacia hidrográfica do rio Moxotó-PE. Revista Brasileira de Geografia Física, v.1, p.55-69, 2010.

SILVA, B.B. da; BRAGA, A.C.; BRAGA, C.C. Balanço de radiação no perímetro irrigado São Gonçalo - $\mathrm{PB}$ mediante imagens orbitais. Revista Caatinga, v.24, p.145-152, 2011.

SILVA, B.B. da; LOPES, G.M.; AZEVEDO, P.V. de. Balanço de radiação em áreas irrigadas utilizando imagens LANDSAT 5 - TM. Revista Brasileira de Meteorologia, v.20, p.243-252, 2005a. 
SILVA, B.B. da; LOPES, G.M.; AZEVEDO, P.V. de. Determinação do albedo de áreas irrigadas com base em imagens LANDSAT 5-TM. Revista Brasileira de Agrometeorologia, v.13, p.201-211, 2005b.

SILVA, B.B. da; SILVA, S.T.A. da; GOMES, H.B. Alterações climáticas decorrentes de mudanças no uso da terra mediante sensoriamento remoto. Mercator, v.9, p.91-106, 2010.
SILVA, R.M. da; SILVA, L.P. e; MONTENEGRO, S.M.G.L.; SANTOS, C.A.G. Análise da variabilidade espaço-temporal e identificação do padrão da precipitação na Bacia do Rio Tapacurá, Pernambuco. Sociedade e Natureza, v.22, p.357-372, 2010a.

SILVA, S.R. da (Coord.). Atlas de bacias hidrográficas de Pernambuco. Recife: Secretaria de Ciência, Tecnologia e Meio Ambiente, 2006. 104p.

Recebido em 23 de julho de 2011 e aprovado em 24 de agosto de 2012 